PROCEEDINGS OF THE

AMERICAN MATHEMATICAL SOCIETY

Volume 137, Number 1, January 2009, Pages 123-134

S 0002-9939(08)09655-X

Article electronically published on August 28, 2008

\title{
ON THE SPECTRUM OF FREQUENTLY HYPERCYCLIC OPERATORS
}

\author{
STANISLAV SHKARIN
}

(Communicated by Marius Junge)

\begin{abstract}
A bounded linear operator $T$ on a Banach space $X$ is called frequently hypercyclic if there exists $x \in X$ such that the lower density of the set $\left\{n \in \mathbb{N}: T^{n} x \in U\right\}$ is positive for any non-empty open subset $U$ of $X$. Bayart and Grivaux have raised a question whether there is a frequently hypercyclic operator on any separable infinite dimensional Banach space. We prove that the spectrum of a frequently hypercyclic operator has no isolated points. It follows that there are no frequently hypercyclic operators on all complex and on some real hereditarily indecomposable Banach spaces, which provides a negative answer to the above question.
\end{abstract}

\section{INTRODUCTION}

All vector spaces in this article are assumed to be over the field $\mathbb{K}, \mathbb{K}$ being either the field $\mathbb{C}$ of complex numbers or the field $\mathbb{R}$ of real numbers. As usual, $\mathbb{T}=\{z \in \mathbb{C}:|z|=1\}, \mathbb{Z}$ is the set of integers, $\mathbb{Z}_{+}$is the set of non-negative integers and $\mathbb{N}$ is the set of positive integers. For a Banach space $X, L(X)$ stands for the algebra of bounded linear operators on $X$ and $X^{*}$ is the space of continuous linear functionals on $X$.

Recall that a continuous linear operator $T$ on a topological vector space $X$ is called hypercyclic if there is an $x \in X$ such that the orbit $\left\{T^{n} x: x \in \mathbb{Z}_{+}\right\}$is dense in $X$. We refer to surveys [17, 18] for additional information on hypercyclicity. There are several stronger versions of the above property of linear operators like hereditarily hypercyclic operators, topologically mixing operators and operators satisfying the Kitai Criterion. Recently Bayart and Grivaux [5] have introduced the concept of frequently hypercyclic operators. Recall that the upper and lower densities of a set $A \subset \mathbb{Z}_{+}$are defined by the formulas

$$
\underline{d}(A)=\varliminf_{n \rightarrow \infty} \frac{|\{m \in A: m \leqslant n\}|}{n}, \quad \bar{d}(A)=\varlimsup_{n \rightarrow \infty} \frac{|\{m \in A: m \leqslant n\}|}{n},
$$

where $|B|$ stands for the number of elements of a finite set $B$.

Received by the editors July 26, 2007.

2000 Mathematics Subject Classification. Primary 47A16, 37A25.

Key words and phrases. Frequently hypercyclic operators, hereditarily indecomposable Banach spaces, quasinilpotent operators.

Partially supported by Plan Nacional I+D+I grant No. MTM2006-09060 and Junta de Andalucía FQM-260.

(C)2008 American Mathematical Society Reverts to public domain 28 years from publication 
Definition 1. Let $T$ be a continuous linear operator on a topological vector space $X$. Then $x \in X$ is called a frequently hypercyclic vector for $T$ if the lower density of the set $\left\{n \in \mathbb{N}: T^{n} x \in U\right\}$ is positive for any non-empty open subset $U$ of $X$. An operator $T$ is called frequently hypercyclic if it has a frequently hypercyclic vector.

We also say that $x \in X$ is a $\mathfrak{U}$-frequently hypercyclic vector for $T$ if the upper density of the set $\left\{n \in \mathbb{N}: T^{n} x \in U\right\}$ is positive for any non-empty open subset $U$ of $X$. An operator $T$ is called $\mathfrak{U}$-frequently hypercyclic if it has a $\mathfrak{U}$-frequently hypercyclic vector. We denote the set of $\mathfrak{U}$-frequently hypercyclic vectors for $T$ by the symbol $\mathfrak{u}(T)$.

Clearly frequent hypercyclicity implies $\mathfrak{U}$-frequent hypercyclicity, which, in turn, implies hypercyclicity. Bayart and Grivaux [5] have raised the following question.

Question 1. Does every separable infinite dimensional Banach space support a frequently hypercyclic operator?

This question is partially motivated by the following chain of results. Ansari [1] and Bernal-González [6] showed independently that for any separable infinite dimensional Banach space $X$ there is a hypercyclic operator $T \in L(X)$. Grivaux [15. proved that there is a mixing operator on any separable infinite dimensional Banach space. Finally, in 25] it is shown that on any separable infinite dimensional Banach space, there is a bounded linear operator, satisfying the Kitai Criterion. In this article we show that unlike for other strong forms of hypercyclicity, the answer to the above question is negative.

Theorem 1.1. Let $T$ be a bounded linear operator on a separable infinite dimensional Banach space $X$ such that $\lim _{n \rightarrow \infty}\left\|T^{n}\right\|^{1 / n}=0$. Then $I+T$ is not $\mathfrak{U}$-frequently hypercyclic. In particular, $I+T$ is not frequently hypercyclic.

In the case $\mathbb{K}=\mathbb{C}$, we use the above theorem to prove the following result.

Theorem 1.2. Let $X$ be a complex Banach space and $T \in L(X)$ be frequently hypercyclic. Then the spectrum $\sigma(T)$ of $T$ has no isolated points.

This result shows that the class of frequently hypercyclic operators is significantly smaller than the class of hypercyclic operators. For instance, in [16 it is demonstrated that if $X$ is a separable Banach space and $M$ is the operator norm closure of the set of finite rank nilpotent operators, then the set $\{T \in M: I+T$ is hypercyclic $\}$ is a dense $G_{\delta}$ subset of $M$. Thus, in a sense, a 'generic' operator with one-point spectrum $\{1\}$ is hypercyclic.

We use Theorem 1.1 to obtain a negative answer to Question 1. It turns out that there are no frequently hypercyclic operators on Banach spaces with few operators. Recall that a bounded linear operator $T$ on a Banach space $X$ is called strictly singular if the restriction of $T$ to any closed infinite dimensional subspace of $X$ is not an isomorphism onto its image. As is well-known [24, the set $\mathcal{S}(X)$ of strictly singular operators is an ideal in the algebra $L(X)$.

By $\Phi_{\mathbb{K}}$ we denote the class of infinite dimensional Banach spaces $X$ over the field $\mathbb{K}$ for which $L(X) / \mathcal{S}(X)$ is isomorphic to $\mathbb{K}$. It is straightforward to see that $X \in \Phi_{\mathbb{K}}$ if and only if any $T \in L(X)$ has the shape $T=z I+S$ for $z \in \mathbb{K}$ and $S \in \mathcal{S}(X)$.

Theorem 1.3. Let $X \in \Phi_{\mathbb{K}}$. Then there is no bounded $\mathfrak{u}$-frequently hypercyclic operator on $X$. In particular, $X$ does not support a frequently hypercyclic operator. 
The above theorem gives a negative answer to Question 1 provided there are separable Banach spaces in classes $\Phi_{\mathbb{C}}$ and $\Phi_{\mathbb{R}}$. Recall that an infinite dimensional Banach space $X$ is called hereditarily indecomposable or an HI space if for any infinite dimensional closed linear subspaces $L$ and $M$ of $X$ satisfying $L \cap M=\{0\}$ their sum $L+M$ is not closed. Separable HI Banach spaces were first constructed by Gowers and Maurey [14; see also the survey [4. One of the most interesting features of HI spaces $X$ is 14,12 that any complex HI space belongs to $\Phi_{\mathbb{C}}$. It is also worth noting that there are separable Banach spaces in $\Phi_{\mathbb{C}}$ that are not HI [3]. Anyway, we have the following corollary.

Corollary 1.4. Let $X$ be a separable complex HI space. Then there is no bounded $\mathcal{U}$-frequently hypercyclic operator on $X$. In particular, $X$ does not support a frequently hypercyclic operator.

The case of real HI spaces is slightly more complicated. Namely, Ferenczi [12, 13 , demonstrated that if $X$ is a real HI space, then $L(X) / \mathcal{S}(X)$ is isomorphic to either $\mathbb{R}$, or $\mathbb{C}$ or the quaternion division ring $\mathbb{H}$. Moreover, in [13] it is shown that all three possibilities do occur on the level of separable real HI spaces and it is observed that the original real separable HI space $X$ of Gowers and Maurey [14] satisfies $L(X) / \mathcal{S}(X) \simeq \mathbb{R}$ and therefore belongs to $\Phi_{\mathbb{R}}$. This remark together with Theorem 1.3 gives us the following corollary.

Corollary 1.5. There is an infinite dimensional separable real Banach space $X$, which does not support a $\mathfrak{U}$-frequently hypercyclic operator. In particular, $X$ does not support a frequently hypercyclic operator.

\section{Proof of Theorem 1.1}

Theorem 2.1. Let $T$ be a continuous linear operator on a real topological vector space $X$ and $x \in X$ be such that there exists $f \in X^{*} \backslash\{0\}$ satisfying

$$
\lim _{n \rightarrow \infty}\left|f\left((T-I)^{n} x\right)\right|^{1 / n}=0 \quad \text { for each } \quad x \in X .
$$

Then $x$ is not a $\mathfrak{U}$-frequently hypercyclic vector for $T$.

Proof. Assume that $x \in \mathfrak{u}(T)$. First, observe, that we can find $u \in X$ such that $f(u)<0$ and $f(T u)>0$. Indeed, otherwise, using continuity of $f$ and $T$, we see that $f(T u) \leqslant 0$ if $f(u) \leqslant 0$. Since $f(-y)=-f(y)$ for any $y \in X$, we also have $f(T u) \geqslant 0$ if $f(u) \geqslant 0$. Hence $T(H) \subseteq H$, where $H=\operatorname{ker} f$. Thus, $T$ has a closed invariant subspace of codimension 1 . On the other hand, in 26 it is proven that any hypercyclic operator on any real or complex topological vector space has no closed invariant subspaces of finite codimension. This contradiction shows that there is $u \in X$ such that $f(u)<0$ and $f(T u)>0$. Since $f$ and $T$ are continuous, we can choose an open neighborhood $U$ of $u$ such that

$$
f(v)<0 \text { and } f(T v)>0 \text { for any } v \in U .
$$

Since $x \in \mathfrak{U}(T)$, there exists a subset $A$ of $\mathbb{Z}_{+}$of positive upper density such that

$$
f\left(T^{n} x\right) \in U \text { for any } n \in A \text {. }
$$

Consider now the series

$$
F(z)=f(x)+\sum_{n=1}^{\infty} \frac{f\left((T-I)^{n} x\right)}{n !} z(z-1) \ldots(z-n+1), \quad z \in \mathbb{C} .
$$


Condition (2.1) implies that the series in (2.4) is uniformly convergent on any compact subset of $\mathbb{C}$ and therefore defines an entire function $F$. Moreover, $F$ has exponential type 0 , that is,

$$
\lim _{R \rightarrow \infty} \frac{\ln M_{F}(R)}{R}=0, \text { where } M_{F}(R)=\max _{|z| \leqslant R}|F(z)| .
$$

Indeed, by (2.1), for any $\varepsilon \in(0,1)$, there is an $m \in \mathbb{N}$ such that $\left|f\left((T-I)^{n} x\right)\right|<\varepsilon^{n}$ for $n>m$. Then

$$
\begin{aligned}
M_{F}(R) & \leqslant|f(x)|+\sum_{n=1}^{\infty} \frac{\left|f\left((T-I)^{n} x\right)\right|}{n !} R(R+1) \ldots(R+n-1) \\
& \leqslant 1+\sum_{n=1}^{\infty} \frac{\varepsilon^{n}}{n !} R(R+1) \ldots(R+n-1)+O\left(R^{m}\right)=(1-\varepsilon)^{-R}+O\left(R^{m}\right) \\
& =e^{a(\varepsilon) R}+O\left(R^{m}\right)=O\left(e^{a(\varepsilon) R}\right) \text { as } R \rightarrow \infty, \text { where } a(\varepsilon)=-\ln (1-\varepsilon) .
\end{aligned}
$$

Since $a(\varepsilon) \rightarrow 0$ as $\varepsilon \rightarrow 0$, (2.5) follows. From the definition of $F$ it also easily follows that

$$
F(k)=f\left(T^{k} x\right) \text { for any } k \in \mathbb{Z}_{+} .
$$

Using (2.6), (2.3) and (2.2), we see that $F(n)<0$ and $F(n+1)>0$ for any $n \in A$. Since $F$ is real on the real axis, for any $n \in A$, there is $t_{n} \in(n, n+1)$ such that $F\left(t_{n}\right)=0$. Now let $N_{F}(R)$ be the number of zeros of $F$ in the set $\{z \in \mathbb{C}: 0<|z|<R\}$. The last observation yields $N_{F}(n+1) \geqslant k_{n}$ for any $n \in \mathbb{Z}_{+}$, where $k_{n}=|\{m \in A: m \leqslant n\}|$ is the counting function of the set $A$. On the other hand, by a corollary of Jensen's Theorem (see [23, p. 15]) we have

$$
N_{F}(R) \leqslant \ln M_{F}(e R)+O(\ln R) \quad \text { as } R \rightarrow \infty .
$$

Hence

$$
\varlimsup_{n \rightarrow \infty} \frac{\ln M_{F}(e(n+1))}{n} \geqslant \varlimsup_{n \rightarrow \infty} \frac{N_{F}(n+1)}{n} \geqslant \varlimsup_{n \rightarrow \infty} \frac{k_{n}}{n}=\bar{d}(A)>0,
$$

which contradicts (2.5).

Corollary 2.2. Let $X$ be a Banach space and $T \in L(X)$ be such that there exists $f \in X^{*} \backslash\{0\}$ for which $\lim _{n \rightarrow \infty}\left\|T^{* n} f\right\|^{1 / n}=0$. Then $I+T$ is not $\mathfrak{U}$-frequently hypercyclic.

Proof. If $\mathbb{K}=\mathbb{R}$, we just apply Theorem 2.1 to the operator $I+T$ to see that the latter has no $\mathfrak{U}$-frequently hypercyclic vectors. If $\mathbb{K}=\mathbb{C}$, we consider $X$ as a real Banach space, $T$ as an $\mathbb{R}$-linear operator and apply Theorem 2.1 to the operator $I+T$ and the functional $\operatorname{Re} f$.

Theorem 1.1 follows immediately from Corollary 2.2 since $\left\|T^{* n} f\right\| \leqslant\|f\|\left\|T^{* n}\right\|=$ $\|f\|\left\|T^{n}\right\|$ for any $f \in X^{*}$ and any $n \in \mathbb{Z}_{+}$.

\section{Rotations AND POWERS OF $\mathfrak{U}$-FREQUENTLY HYPERCYCLIC OPERATORS}

In order to reduce Theorem 1.3 and Theorem 1.2 to Theorem 1.1 , we need to show that the statement of Theorem 1.1 remains true if we replace $I+T$ by $z I+T$ for any $z \in \mathbb{K}$ with $|z|=1$. In order to do so, we need to demonstrate that the class of $\mathfrak{U}$-frequently hypercyclic operators is stable under multiplication by scalars $z$ with $|z|=1$. 
For hypercyclic operators acting on complex Banach spaces, this kind of stability was proved by León-Saavedra and Müller [22]. In the case $\mathbb{K}=\mathbb{R}$ the fact that $T$ is hypercyclic if and only if $-T$ is hypercyclic follows from the result of Ansari [2], who showed that hypercyclicity of $T$ implies hypercyclicity of its powers. In particular $T^{2}$ is hypercyclic if $T$ is. Since $(-T)^{2}=T^{2}$, the latter observation shows that $T$ is hypercyclic if and only if $-T$ is. We are going to prove the same stability results for $\mathfrak{U}$-frequently hypercyclic operators. Concerning the powers, we shall use a result of Bourdon and Feldman [10, who demonstrated that an orbit of a continuous linear operator on a locally convex topological vector space is either everywhere dense or nowhere dense. The only obstacle that did not allow the same construction to work for arbitrary topological vector spaces was that the proof of the density of the range of $p(T)$ for any non-zero polynomial $p$ and a hypercyclic operator $T$ used the duality argument. This obstacle was removed by Wengenroth [26], which allowed him to extend the Bourdon and Feldman theorem to arbitrary topological vector spaces; see also the survey [18]. Throughout this section $X$ is a topological vector space and $T \in L(X)$.

Theorem A. Let $x \in X$ be such that $F_{x}^{T}=\overline{\left\{T^{n} x: n \in \mathbb{Z}_{+}\right\}}$has non-empty interior. Then $F_{x}^{T}=X$.

Although the above-mentioned result of León-Saavedra and Müller is formulated and proved for operators acting on Banach spaces, the same proof with minor modifications (just replace convergent sequences by convergent nets and use the result of Wengenroth to ensure density of the ranges of polynomials of $T$ instead of the duality argument) gives the following extension of their result.

Theorem B. Let $z \in \mathbb{K},|z|=1$. Then the sets of hypercyclic vectors for $T$ and $z T$ coincide.

For brevity, we denote by $\Lambda$ the set of all subsets of $\mathbb{Z}_{+}$of positive upper density. We also fix a base $\mathcal{U}$ of open neighborhoods of 0 in $X$.

Definition 2. For $x \in X$ we denote

$$
M_{x}^{T}=\left\{y \in X:\left\{n \in \mathbb{Z}_{+}: y-T^{n} x \in U\right\} \in \Lambda \text { for any } U \in \mathcal{U}\right\} .
$$

Clearly, $x \in \mathfrak{U}(T)$ if and only if $M_{x}^{T}=X$.

If additionally $X$ is complex, we denote

$$
\begin{aligned}
& N_{x}^{T}=\left\{y \in X:\left\{n \in \mathbb{Z}_{+}: C_{n}(T, x) \cap(y+U) \neq \varnothing\right\} \in \Lambda \text { for any } U \in \mathcal{U}\right\}, \\
& \quad \text { where } C_{n}(T, x)=\left\{z T^{n} x: z \in \mathbb{T}\right\} .
\end{aligned}
$$

We start by establishing a few straightforward properties of the sets $M_{x}^{T}$ and $N_{x}^{T}$ defined in (3.1) and (3.2). The following lemma is an elementary exercise. We leave the proof to the reader.

Lemma 3.1. For any $x \in X, M_{x}^{T}$ is closed in $X, T\left(M_{x}^{T}\right) \subseteq M_{x}^{T}, M_{T x}^{T}=M_{x}^{T}$ and $M_{z x}^{T}=z M_{x}^{T}$ for each $z \in \mathbb{K}$. If $X$ is complex, then for any $x \in X, N_{x}^{T}$ is closed in $X, T\left(N_{x}^{T}\right) \subseteq N_{x}^{T}, N_{T x}^{T}=N_{x}^{T}$ and $N_{z x}^{T}=N_{x}^{z T}=N_{x}^{T}$ for any $z \in \mathbb{T}$.

Lemma 3.2. If $u, x, y \in X$ are such that $y \in M_{x}^{T}$ and $x \in M_{u}^{T}$, then $y \in M_{u}^{T}$.

Proof. Let $U \in \mathcal{U}$. Pick $V \in \mathcal{U}$ such that $V+V \subset U$. Since $y \in M_{x}^{T}$, we can find $m \in \mathbb{Z}_{+}$such that $y-T^{m} x \in V$. Since $x \in M_{u}^{T}$, we have $A=\left\{k \in \mathbb{Z}_{+}: x-T^{k} u \in\right.$ 
$\left.\left(T^{m}\right)^{-1}(V)\right\} \in \Lambda$. Then for any $k \in A$, we have

$$
y-T^{k+m} u=y-T^{m} x+T^{m}\left(x-T^{k} u\right) \in V+T^{m}\left(\left(T^{m}\right)^{-1}(V)\right) \subseteq V+V \subseteq U .
$$

Thus $y-T^{n} u \in U$ for any $n \in m+A$. Since $m+A \in \Lambda$, we have $y \in M_{u}^{T}$.

Lemma 3.3. If $X$ is complex and $x \in N_{x}^{T}$, then $x \in M_{x}^{T}$.

Proof. Clearly the set $F=\left\{\mu \in \mathbb{T}: \mu x \in M_{x}^{T}\right\}$ is closed since $M_{x}^{T}$ is. Let us verify that $F$ is non-empty. For each $U \in \mathcal{U}$ consider

$$
F(U)=\left\{z \in \mathbb{T}:\left\{n \in \mathbb{Z}_{+}: z x-T^{n} x \in U\right\} \in \Lambda\right\}
$$

and let $\bar{F}(U)$ be the closure of $F(U)$ in $\mathbb{T}$. Clearly $F=\bigcap\{F(U): U \in \mathcal{U}\}$. Let us show that the sets $F(U)$ are non-empty. Fix $U \in \mathcal{U}$. Choose $V \in \mathcal{U}$ and $\varepsilon>0$ such that $V+D_{\varepsilon} x \subseteq U$, where $D_{\varepsilon}=\{z \in \mathbb{C}:|z|<\varepsilon\}$. Since $x \in N_{x}^{T}$, there exist $A \in \Lambda$ and a sequence $\left\{z_{m}\right\}_{m \in A}$ of elements of $\mathbb{T}$ such that $z_{m} x-T^{m} x \in V$ for any $m \in A$. Let $k \in \mathbb{N}$ be such that $k>\varepsilon^{-1}$ and let $w_{j}=e^{2 \pi i j / k}$ for $0 \leqslant j \leqslant k-1$. Then for any $m \in A$ we can pick $\nu(m) \in\{0, \ldots, k-1\}$ such that $\left|z_{m}-w_{\nu(m)}\right|<\varepsilon$. Clearly $A$ is the union of $A_{j}=\{m \in A: \nu(m)=j\}$ for $0 \leqslant j \leqslant k-1$. Since the union of $A_{j}$ has positive upper density, there exists $j \in\{0, \ldots, k-1\}$ such that $A_{j} \in \Lambda$. For each $m \in A_{j}$, we have

$$
w_{j} x-T^{m} x=z_{m} x-T^{m} x+\left(w_{j}-z_{m}\right) x \in V+D_{\varepsilon} x \subset U .
$$

Hence $w_{j} \in F(U)$ and $F(U)$ is non-empty. From the definition of the set $F(U)$, we also have that $\bar{F}(V) \subset J_{\varepsilon} F(V) \subseteq F(U)$, where $J_{\varepsilon}=\left\{e^{i t}:-\varepsilon \leqslant t \leqslant \varepsilon\right\}$. Thus for each $U \in \mathcal{U}$ there is $V \in \mathcal{U}$ such that $\bar{F}(V) \subset F(U)$. It follows that

$$
F=\bigcap_{U \in \mathcal{U}} F(U)=\bigcap_{U \in \mathcal{U}} \bar{F}(U) .
$$

Finally, for any $U_{1}, \ldots, U_{n} \in \mathcal{U}$, we have

$$
\varnothing \neq F\left(U_{1} \cap \ldots \cap U_{n}\right) \subseteq F\left(U_{1}\right) \cap \ldots \cap F\left(U_{n}\right) \subseteq \bar{F}\left(U_{1}\right) \cap \ldots \cap \bar{F}\left(U_{n}\right) .
$$

Thus $\{\bar{F}(U): U \in \mathcal{U}\}$ is a family of closed subsets of the compact topological space $\mathbb{T}$, any finite subfamily of which has non-empty intersection. Hence it has non-empty intersection and (3.3) implies that $F \neq \varnothing$.

Thus there is $z \in \mathbb{T}$ such that $z x \in M_{x}^{T}$. By Lemma 3.1, then $z^{2} x \in M_{z x}^{T}$. According to Lemma 3.2 the inclusions $z x \in M_{x}^{T}$ and $z^{2} x \in M_{z x}^{T}$ imply $z^{2} x \in M_{x}^{T}$. Proceeding inductively, we have $\left\{z^{n} x: n \in \mathbb{N}\right\} \subset M_{x}^{T}$. That is, $Q_{z}=\left\{z^{n}: n \in\right.$ $\mathbb{N}\} \subseteq F$. Since $F$ is closed, $\overline{Q_{z}} \subseteq F$. Since $1 \in \overline{Q_{z}}$ for any $z \in \mathbb{T}$, we see that $1 \in F$. That is, $x \in M_{x}^{T}$.

Lemma 3.4. If $x \in X$ is such that $x \in M_{x}^{T}$, then $M_{x}^{T}=F_{x}^{T}$, where $F_{x}^{T}=$ $\overline{\left\{T^{n} x: n \in \mathbb{Z}_{+}\right\}}$.

Proof. Clearly $M_{x}^{T} \subseteq F_{x}^{T}$. Since $x \in M_{x}^{T}$ and $T\left(M_{x}^{T}\right) \subseteq M_{x}^{T}$, we see that $\left\{T^{n} x\right.$ : $\left.n \in \mathbb{Z}_{+}\right\} \subseteq M_{x}^{T}$. Since $M_{x}^{T}$ is closed, we have $F_{x}^{T} \subseteq M_{x}^{T}$. Thus, $F_{x}^{T}=M_{x}^{T}$.

Lemma 3.5. If $x \in X$ is such that $M_{x}^{T}$ has non-empty interior, then there exists $y \in X$ such that $y \in M_{y}^{T}=M_{x}^{T}$.

Proof. Since $M_{x}^{T}$ has non-empty interior, there is $k \in \mathbb{Z}_{+}$such that $T^{k} x$ is an interior point of $M_{x}^{T}$. In particular, $y=T^{k} x \in M_{x}^{T}$. By Lemma 3.1, $M_{y}^{T}=$ $M_{T^{k} x}^{T}=M_{x}^{T}$. Thus $y \in M_{x}^{T}=M_{y}^{T}$. 


\subsection{Rotations.}

Proposition 3.6. Let $X$ be a complex topological vector space, $z \in \mathbb{T}$ and $T \in$ $L(X)$. Then $T$ is $\mathfrak{U}$-frequently hypercyclic if and only if $z T$ is $\mathfrak{U}$-frequently hypercyclic and $\mathfrak{U}(T)=\mathfrak{U}(z T)$.

Proof. Let $x \in \mathfrak{U}(T)$. Then $M_{x}^{T}=N_{x}^{T}=X$, where the sets $M_{x}^{T}$ and $N_{x}^{T}$ are defined in (3.1) and (3.2). On the other hand, clearly $N_{x}^{z T}=N_{x}^{T}$. Hence $N_{x}^{z T}=X$. In particular, $x \in N_{x}^{z T}$. By Lemma 3.3, $x \in M_{x}^{z T}$. Then by Lemma 3.4, $M_{x}^{z T}=F_{x}^{z T}$, where $F_{x}^{z T}$ is the closure of $\left\{(z T)^{n} x: n \in \mathbb{Z}_{+}\right\}$. Since $x$ is a hypercyclic vector for $T$, Theorem B implies that $x$ is a hypercyclic vector for $z T$ and therefore $F_{x}^{z T}=X$. Hence $M_{x}^{z T}=X$ and $x \in \mathfrak{U}(z T)$. Thus $\mathfrak{U}(T) \subseteq \mathfrak{U}(z T)$. Applying this inclusion to $T$ replaced by $z T$ and $z$ replaced by $z^{-1}$, we obtain $\mathfrak{u}(z T) \subseteq \mathfrak{u}(T)$. Thus $\mathfrak{u}(T)=\mathfrak{u}(z T)$.

\subsection{Powers.}

Proposition 3.7. Let $X$ be a topological vector space and $T \in L(X)$. If $x \in X$ is such that the set $M_{x}^{T}$, defined in (3.1), has non-empty interior, then $x \in \mathfrak{U}(T)$.

Proof. By Lemma 3.5, we can pick $y \in X$ such that $y \in M_{y}^{T}=M_{x}^{T}$. By Lemma 3.4. $M_{y}^{T}=F_{y}^{T}$, where $F_{y}^{T}$ is the closure of $\left\{T^{n} y: n \in \mathbb{Z}_{+}\right\}$. Thus, $F_{y}^{T}$ has non-empty interior and Theorem A implies that $F_{y}^{T}=X$. Since $F_{y}^{T}=M_{y}^{T}=M_{x}^{T}$, we have $M_{x}^{T}=X$. That is, $x \in \mathfrak{U}(T)$.

Corollary 3.8. Let $X$ be a topological vector space, $T \in L(X)$ be a $\mathfrak{U}$-frequently hypercyclic operator and $n \in \mathbb{N}$. Then $T^{n}$ is also $\mathfrak{U}$-frequently hypercyclic. Moreover $\mathfrak{u}(T)=\mathfrak{U}\left(T^{n}\right)$.

Proof. Let $x \in \mathfrak{U}(T)$. Then the set $M_{x}^{T}$ defined in (3.1) coincides with $X$. Since the union of finitely many subsets of $\mathbb{Z}_{+}$has positive upper density if and only if at least one of them has positive upper density, one can easily see that

$$
X=M_{x}^{T}=\bigcup_{j=0}^{n-1} M_{T^{j} x}^{T^{n}} .
$$

By Lemma 3.1. the sets $M_{T^{j} x}^{T^{n}}$ are closed. Hence there is $j \in\{0, \ldots, n-1\}$ such that $M_{T^{j} x}^{T^{n}}$ has non-empty interior. By Proposition 3.7. $M_{T^{j} x}^{T^{n}}=X$. That is, $T^{j} x \in \mathfrak{u}\left(T^{n}\right)$. Exactly as for the usual hypercyclicity, one can easily verify that if $y$ is a $\mathfrak{U}$-frequently hypercyclic vector for $S \in L(X)$ and $R \in L(X)$ has dense range and commutes with $S$, then $R y \in \mathfrak{U}(S)$. Applying this observation with $S=T^{n}$, $y=T^{j} x$ and $R=T^{n-j}$, we see that $T^{n} x \in \mathfrak{u}\left(T^{n}\right)$. Since $x$ and $T^{n} x$ have the same orbits with respect to $T^{n}$ (up to one element added or removed), we obtain $x \in \mathfrak{U}\left(T^{n}\right)$. Thus $\mathfrak{U}(T) \subseteq \mathfrak{U}\left(T^{n}\right)$. The inclusion $\mathfrak{U}\left(T^{n}\right) \subseteq \mathfrak{U}(T)$ is obvious.

Corollary 3.9. Let $X$ be a real topological vector space and $T \in L(X)$. Then $T$ is $\mathfrak{U}$-frequently hypercyclic if and only if $-T$ is $\mathfrak{U}$-frequently hypercyclic and $\mathfrak{U}(T)=$ $\mathfrak{u}(-T)$.

Proof. By Corollary 3.8, $\mathfrak{u}(T)=\mathfrak{u}\left(T^{2}\right)=\mathfrak{u}\left((-T)^{2}\right)=\mathfrak{u}(-T)$. 


\section{Proof of Theorems 1.3 and 1.2}

The above results allow us to strengthen Theorem 1.1

Corollary 4.1. Let $X$ be a separable infinite dimensional Banach space, $z \in \mathbb{K}$ and $T \in L(X)$ be such that $|z|=1$ and there exists $f \in X^{*} \backslash\{0\}$ for which $\lim _{n \rightarrow \infty}\left\|T^{* n} f\right\|^{1 / n}=0$. Then $z I+T$ is not $\mathfrak{U}$-frequently hypercyclic.

Proof. By Corollary 2.2 $I+z^{-1} T$ is not $\mathfrak{U}$-frequently hypercyclic. Using Proposition $[3.6$ in the case $\mathbb{K}=\mathbb{C}$ and Corollary 3.9 in the case $\mathbb{K}=\mathbb{R}$, we see that $z\left(I+z^{-1} T\right)=z I+T$ is not $\mathfrak{U}$-frequently hypercyclic.

Since $\left\|T^{* n} f\right\| \leqslant\|f\|\left\|T^{* n}\right\|=\|f\|\left\|T^{n}\right\|$ for any $f \in X^{*}$ and any $n \in \mathbb{Z}_{+}$, we immediately obtain the following slightly stronger form of Theorem 1.1.

Theorem 4.2. Let $T$ be a bounded linear operator on a separable infinite dimensional Banach space $X$ such that $\lim _{n \rightarrow \infty}\left\|T^{n}\right\|^{1 / n}=0$ and $z \in \mathbb{K}$ be such that $|z|=1$. Then $z I+T$ is not $\mathfrak{U}$-frequently hypercyclic. In particular, $z I+T$ is not frequently hypercyclic.

In order to prove Theorems 1.3 and 1.2, we need the following lemmas.

Lemma 4.3. Let $X \in \Phi_{\mathbb{K}}$ and $T \in L(X)$. Assume also that $T$ has no non-trivial closed invariant subspaces of finite codimension. Then $T=z I+S$, where $z \in \mathbb{K}$ and $\lim _{n \rightarrow \infty}\left\|S^{n}\right\|^{1 / n}=0$.

Proof. Since $X \in \Phi_{\mathbb{K}}$, there are $z \in \mathbb{K}$ and $S \in \mathcal{S}(X)$ such that $T=z I+S$. Let $X_{\mathbb{C}}=X$ and $R_{\mathbb{C}}=R$ for any $R \in L(X)$ if $\mathbb{K}=\mathbb{C}$ and let $X_{\mathbb{C}}$ be the complexification of $X$ and $R_{\mathbb{C}} \in L\left(X_{\mathbb{C}}\right)$ be the complexification (= the unique complex linear extension) of $R \in L(X)$ if $\mathbb{K}=\mathbb{R}$.

In any case $T_{\mathbb{C}}=z I+S_{\mathbb{C}}$ and $S_{\mathbb{C}} \in \mathcal{S}(X)$. The fact that $T$ has no non-trivial closed linear subspaces of finite codimension is clearly equivalent to the statement that the point spectrum $\sigma_{p}\left(T_{\mathbb{C}}^{*}\right)$ of the dual $T_{\mathbb{C}}^{*}$ of $T_{\mathbb{C}}$ is empty.

Assume that $w \in \sigma\left(T_{\mathbb{C}}\right) \backslash\{z\}$. Then $w-z \in \sigma\left(S_{\mathbb{C}}\right) \backslash\{0\}$. Since a non-zero element of the spectrum of any strictly singular operator on a complex Banach space is a normal eigenvalue [24], we see that $w-z$ is a normal eigenvalue of $S_{\mathbb{C}}$ and therefore $w-z \in \sigma_{p}\left(S_{\mathbb{C}}^{*}\right)$. Hence $w \in \sigma_{p}\left(T_{\mathbb{C}}^{*}\right)$, which contradicts the equality $\sigma_{p}\left(T_{\mathbb{C}}^{*}\right)=\varnothing$. Thus $\sigma\left(T_{\mathbb{C}}\right)=\{z\}$. Hence $\sigma\left(S_{\mathbb{C}}\right)=\{0\}$. By the spectral radius formula $\lim _{n \rightarrow \infty}\left\|S_{\mathbb{C}}^{n}\right\|^{1 / n}=0$. Since $S$ is the restriction of $S_{\mathbb{C}}$ to an $\mathbb{R}$-linear invariant subspace, $\left\|S^{n}\right\| \leqslant\left\|S_{\mathbb{C}}^{n}\right\|$ for any $n \in \mathbb{N}$ and therefore $\lim _{n \rightarrow \infty}\left\|S^{n}\right\|^{1 / n}=0$.

Lemma 4.4. Let $X \in \Phi_{\mathbb{K}}$ and $T \in L(X)$ be a hypercyclic operator. Then $T=$ $z I+S$, where $z \in \mathbb{K},|z|=1$ and $\lim _{n \rightarrow \infty}\left\|S^{n}\right\|^{1 / n}=0$.

Proof. As is shown in 26], a hypercyclic operator on a real or complex topological vector space has no non-trivial closed invariant subspaces of finite codimension. Hence, by Lemma 4.3. $T=z I+S$, where $z \in \mathbb{K}$ and $\lim _{n \rightarrow \infty}\left\|S^{n}\right\|^{1 / n}=0$. It remains to demonstrate that $|z|=1$. Using the equalities $T=z I+S$ and $\lim _{n \rightarrow \infty}\left\|S^{n}\right\|^{1 / n}=0$, one can easily verify that

$$
\lim _{n \rightarrow \infty}\left\|T^{n} x\right\|^{1 / n}=|z| \text { for any } x \in X \backslash\{0\} .
$$


Now if $|z|>1$, then according to (4.1), $\left\|T^{n} x\right\| \rightarrow \infty$ as $n \rightarrow \infty$ for each non-zero $x \in X$. Similarly, if $|z|<1$, then $\left\|T^{n} x\right\| \rightarrow 0$ as $n \rightarrow \infty$ for any $x \in X$. Hence $T$ cannot be hypercyclic if $|z| \neq 1$. Thus $|z|=1$.

Theorem 1.3 follows immediately from Lemma 4.4 and Theorem 4.2

Proof of Theorem 1.2. Let $T$ be a $\mathfrak{U}$-frequently hypercyclic operator on a complex Banach space $X$. Assume also that $z$ is an isolated point of the spectrum $\sigma(T)$. Let $x \in \mathfrak{U}(T), P$ be the spectral projection corresponding to the component $\{z\}$ of the spectrum of $T, Y=P(X)$ and $S \in L(Y)$ be the restriction of $T$ to the closed invariant subspace $Y$. Clearly $\sigma(S)=\{z\}$. Since $S^{n} P x=P T^{n} x$ for each $n \in \mathbb{Z}_{+}$, we see that $P x \in \mathfrak{U}(S)$. Thus, $S$ is a $\mathfrak{U}$-frequently hypercyclic operator on $Y$ satisfying $\sigma(S)=\{z\}$. If $|z|<1$, then $\left\|S^{n} y\right\| \rightarrow 0$, while $\left\|S^{n} y\right\| \rightarrow \infty$ if $|z|>1$ for any $y \in Y$ and $S$ cannot be hypercyclic. Hence $z \in \mathbb{T}$. Since $\sigma(S)=\{z\}$, we have $S=z I+R$, where $\lim _{n \rightarrow \infty}\left\|R^{n}\right\|^{1 / n}=0$. We have obtained a contradiction with Theorem 4.2 ,

\section{Concluding Remarks}

1. In the proofs of Proposition 3.6 and Corollary 3.8, we use the following property of the family $\Lambda$ of subsets of $\mathbb{Z}_{+}$of positive upper density. Namely, if a finite union of sets belongs to $\Lambda$, then one of them does. The family $\Lambda_{0}$ of subsets of $\mathbb{Z}_{+}$of positive lower density fails to have this property. Thus our proof of stability of the class of $\mathfrak{U}$-frequently hypercyclic operators under powers and under multiplication by unimodular scalars does not work for the class of frequently hypercyclic operators. This leads to the following question.

Question 2. Let $T$ be a frequently hypercyclic operator on a separable infinite dimensional Banach space $X$. Is it true that the operators $T^{n}$ and $z T$ for $n \in \mathbb{N}$ and $z \in \mathbb{K}$ with $|z|=1$ are frequently hypercyclic?

2. Proposition 3.6. Corollary 3.8 and Corollary 3.9 admit the following generalization. Let $\Omega$ be any family of non-empty subsets of $\mathbb{Z}_{+}$satisfying the following conditions:

if $A \in \Omega$ and $A \subseteq B \subseteq \mathbb{Z}_{+}$, then $B \in \Omega$;

if $A \in \Omega$ and $k \in \mathbb{Z}_{+}$, then $A+k \in \Omega$ and $(A-k) \cap \mathbb{Z}_{+} \in \Omega$;

if $k \in \mathbb{N}$ and $A_{j}$ for $1 \leqslant j \leqslant k$ are subsets of $\mathbb{Z}_{+}$such that $\bigcup_{j=1}^{k} A_{j} \in \Omega$, then there exists $j \in\{1, \ldots, k\}$ such that $A_{j} \in \Omega$.

We say that $x$ is an $\Omega$-hypercyclic vector for a continuous linear operator $T$ on a topological vector space $X$ if $\left\{n \in \mathbb{Z}_{+}: T^{n} x \in U\right\} \in \Omega$ for each non-empty open subset $U$ of $X$ and that $T$ is $\Omega$-hypercyclic if it has an $\Omega$-hypercyclic vector.

All proofs in Section 3 work without any changes (just replace $\Lambda$ by $\Omega$ ) for $\Omega$ hypercyclicity instead of $\mathfrak{U}$-frequent hypercyclicity. Thus, the class of $\Omega$-hypercyclic operators is stable under taking the powers and under multiplying by unimodular scalars. Note also that when $\Omega$ is the set of all infinite subsets of $\mathbb{Z}_{+}$, then $\Omega$ hypercyclicity coincides with the usual hypercyclicity. The following example seems to be interesting. Let $\Psi$ be the set of all decreasing sequences $\varphi=\left\{\varphi_{n}\right\}_{n \in \mathbb{Z}_{+}}$of positive numbers such that $\sum_{n=0}^{\infty} \varphi_{n}=\infty$. Clearly, for each $\varphi \in \Psi$, the family $\Omega_{\varphi}$

of subsets $A$ of $\mathbb{Z}_{+}$for which $\sum_{n \in A} \varphi_{n}=\infty$ satisfies all properties from the above 
display. It is also easy to verify that an operator $T$ is $\mathfrak{U}$-frequently hypercyclic if and only if it is $\Omega_{\varphi}$-hypercyclic for any $\varphi \in \Psi$. This naturally leads to the following question.

Question 3. For which $\varphi \in \Psi$ is there an $\Omega_{\varphi}$-hypercyclic operator on any separable infinite dimensional Banach space?

3. One can observe that all operators $T$ proven to be frequently hypercyclic have a stronger property. Namely they admit a vector $x$ such that for any non-empty open set $U$ the set $\left\{n \in \mathbb{Z}_{+}: T^{n} x \in U\right\}$ contains a subset, which has positive density. Since there are sets $A \subset \mathbb{Z}_{+}$of positive lower density which contain no subsets of positive density, the last property of $x$ is, formally speaking, stronger than to be a frequently hypercyclic vector. It might be interesting to find out whether all frequently hypercyclic operators admit vectors having this stronger property.

4. Herrero 20] has characterized the operator norm closure $\overline{\mathrm{HC}(\mathcal{H})}$ of the set $\mathrm{HC}(\mathcal{H})$ of hypercyclic operators on a separable infinite dimensional complex Hilbert space $\mathcal{H}$. Later Herrero and Wang 21 have shown that any $T \in \overline{\mathrm{HC}(\mathcal{H})}$ is a limit of a sequence $T_{n}$ of elements of $\mathrm{HC}(\mathcal{H})$ such that the differences $T-T_{n}$ are compact. Using these results, one can easily prove the following proposition.

Proposition 5.1. A non-empty compact subset $K$ of $\mathbb{C}$ is the spectrum of some bounded hypercyclic operator $T$ on a separable infinite dimensional complex Hilbert space $\mathcal{H}$ if and only if $K \cup \mathbb{T}$ is connected.

Proof. The 'only if' part is proved in 20. Let $K$ be a non-empty compact subset of $\mathbb{C}$ such that $K \cup \mathbb{T}$ is connected. We have to show that there is a hypercyclic operator $T \in L(\mathcal{H})$ such that $\sigma(T)=K$. Let $A$ be the set of isolated points of $K$. Exactly as in the proof of Theorem 1.2, one can easily see that $A \subset \mathbb{T}$. Clearly $A$ is at most countable. Let $S \in L\left(\ell_{2}\right)$ be a compact weighted backward shift: $S e_{0}=0$ and $S e_{n}=w_{n} e_{n-1}$ for $n \in \mathbb{N}$, where $w=\left\{w_{n}\right\}_{n \in \mathbb{N}}$ is a sequence of non-zero complex numbers tending to 0 as $n \rightarrow \infty$. Consider the $\ell_{2}$-direct sum $T_{0}$ of $z I+S$ for $z \in A$ :

$$
T_{0}=\bigoplus_{z \in A}(z I+S) .
$$

Then $\sigma\left(T_{0}\right)=\bar{A}$. From [16. Theorem 5.1] it follows that $T_{0}$ is topologically mixing. Next, we take a bounded normal operator $S_{1}$ on a separable complex Hilbert space $\mathcal{H}_{1}$ such that $\sigma\left(S_{1}\right)=K \backslash A$ and $S_{1}$ has empty point spectrum. According to the mentioned results of Herrero [20] and Herrero and Wang 21, we have that $S_{1} \in \overline{\mathrm{HC}\left(\mathcal{H}_{1}\right)}$ and therefore there exists a compact operator $K \in L\left(\mathcal{H}_{1}\right)$ such that $T_{1}=S_{1}+K$ is hypercyclic. For each $z \in \mathbb{C} \backslash \sigma\left(S_{1}\right)=\mathbb{C} \backslash(K \backslash A)$, the operator $S_{1}-z I$ is invertible and therefore by the Fredholm theorem, either $T_{1}-z I$ is invertible or $T_{1}^{*}-z I$ has non-trivial kernel (here $T_{1}^{*}$ is the dual operator, not the Hilbert space adjoint). The latter case is not possible since the dual of any hypercyclic operator has empty point spectrum. Thus $\sigma\left(T_{1}\right) \subseteq \sigma\left(S_{1}\right)$. On the other hand, $S_{1}$ has purely continuous spectrum and $T_{1}$ is a compact perturbation of $S_{1}$. Hence $\sigma\left(S_{1}\right) \subseteq \sigma\left(T_{1}\right)$. Thus $\sigma\left(T_{1}\right)=K \backslash A$. Let $T=T_{0} \oplus T_{1}$. Then $\sigma(T)=\sigma\left(T_{0}\right) \cup \sigma\left(T_{1}\right)=\bar{A} \cup(K \backslash A)=K$. Moreover $T$ is hypercyclic as a direct sum of a hypercyclic operator with a mixing operator. 
Question 4. What compact subsets of $\mathbb{C}$ are the spectra of frequently hypercyclic operators on $\ell_{2}$ ? What about $\mathfrak{U}$-frequently hypercyclic operators?

\section{ACKNOWLEDGEMENT}

The author would like to thank the referee for helpful comments and corrections.

\section{REFERENCES}

[1] S. Ansari, Existence of hypercyclic operators on topological vector spaces, J. Funct. Anal. 148 (1997), 384-390. MR1469346 (98h:47028a)

[2] S. Ansari, Hypercyclic and cyclic vectors, J. Funct. Anal. 128 (1995), 374-383. MR1319961 (96h:47002)

[3] S. Argyros and A. Manoussakis, An indecomposable and unconditionally saturated Banach space, Studia Math. 159 (2003), 1-32. MR2030739 (2005d:46022)

[4] S. Argyros and A. Tolias, Methods in the theory of hereditarily indecomposable Banach spaces, Mem. Amer. Math. Soc. 170 (2004), vi + 114 pp. MR2053392 (2005f:46022)

[5] F. Bayart and S. Grivaux, Frequently hypercyclic operators, Trans. Amer. Math. Soc. 358 (2006), 5083-5117. MR.2231886 (2007e:47013)

[6] L. Bernal-González, On hypercyclic operators on Banach spaces, Proc. Amer. Math. Soc. 127 (1999), 1003-1010. MR.1476119 (99f:47010)

[7] J. Bés and A. Peris, Hereditarily hypercyclic operators, J. Funct. Anal. 167 (1999), 94-112. MR 1710637 (2000f:47012)

[8] J. Bonet and A. Peris, Hypercyclic operators on non-normable Fréchet spaces, J. Funct. Anal. 159 (1998), 587-595. MR1658096 (99k:47044)

[9] A. Bonilla and K.-G. Grosse-Erdmann, On a theorem of Godefroy and Shapiro, Integral Equations Operator Theory 56 (2006), 151-162. MR2264513 (2007h:47020)

[10] P. Bourdon and N. Feldman, Somewhere dense orbits are everywhere dense, Indiana Univ. Math. J. 52 (2003), 811-819. MR 1986898(2004d:47020)

[11] M. De La Rosa and C. Read, A hypercyclic operator whose direct sum is not hypercyclic, preprint.

[12] V. Ferenczi, Operators on subspaces of hereditarily indecomposable Banach spaces, Bull. London Math. Soc. 29 (1997), 338-344. MR.1435570 (98b:47028)

[13] V. Ferenczi, Uniqueness of complex structure and real hereditarily indecomposable Banach spaces, Advances Math. 213 (2007), 462-488. MR2331251

[14] W. Gowers and B. Maurey, The unconditional basic sequence problem, J. Amer. Math. Soc. 6 (1993), 851-874. MR1201238 (94k:46021)

[15] S. Grivaux, Hypercyclic operators, mixing operators and the bounded steps problem, J. Operator Theory 54 (2005), 147-168. MR2168865 (2006k:47021)

[16] S. Grivaux and S. Shkarin, Non-mixing hypercyclic operators, preprint.

[17] K.-G. Grosse-Erdmann, Universal families and hypercyclic operators, Bull. Amer. Math. Soc. 36 (1999), 345-381. MR1685272 (2000c:47001)

[18] K.-G. Grosse-Erdmann, Recent developments in hypercyclicity, RACSAM Rev. R. Acad. Cienc. Exactas Fis. Nat. Ser. A Mat. 97 (2003), 273-286. MR2068180 (2005c:47010)

[19] K.-G. Grosse-Erdmann and A. Peris, Frequently dense orbits, C. R. Math. Acad. Sci. Paris 341 (2005), 123-128. MR2153969 (2006a:47017)

[20] D. Herrero, Limits of hypercyclic and supercyclic operators, J. Funct. Anal. 99 (1991), 179190. MR1120920 (92g:47026)

[21] D. Herrero and Z. Wang, Compact perturbations of hypercyclic and supercyclic operators, Indiana Univ. Math. J. 39 (1990), 819-829. MR.1078739 (91k:47042)

[22] F. León-Saavedra and Vladimir Müller, Rotations of hypercyclic and supercyclic operators, Integral Equations and Operator Theory 50 (2004), 385-391. MR2104261 (2005g:47009)

[23] B. Levin, Distribution of zeros of entire functions, Amer. Math. Soc., Providence, Rhode Island, 1980. MR $589888(81 \mathrm{k}: 30011)$

[24] A. Pietsch, Operator ideals, North-Holland, Amsterdam, 1980. MR582655 (81j:47001) 
[25] S. Shkarin, The Kitai criterion and backward shifts, Proc. Amer. Math. Soc. 136 (2008), 1659-1670. MR 2373595

[26] J. Wengenroth, Hypercyclic operators on non-locally convex spaces, Proc. Amer. Math. Soc. 131 (2003), 1759-1761. MR.1955262 (2003j:47007)

Department of Pure Mathematics, Queens's University Belfast, University Road, Belfast, BT7 1NN, United Kingdom

E-mail address: s.shkarin@qub.ac.uk 